\title{
The Prognostic Significance of CD44V6, CDH11, and $\beta$-Catenin Expression in Patients with Osteosarcoma
}

\author{
Zhouming Deng, ${ }^{1}$ Guangfeng Niu, ${ }^{2}$ Lin Cai, ${ }^{1}$ Renxiong Wei, ${ }^{1}$ and Xiaolei Zhao ${ }^{1}$ \\ ${ }^{1}$ Department of Orthopedics, Zhongnan Hospital of Wuhan University, No. 169 Donghu Road, Wuhan, Hubei Province 430071, China \\ ${ }^{2}$ Department of Spine, Shandong Provincial Hospital, Jinan 250021, China
}

Correspondence should be addressed to Lin Cai; guke3559@aliyun.com

Received 29 April 2013; Revised 1 July 2013; Accepted 1 July 2013

Academic Editor: Christian Mühlfeld

Copyright ( 2013 Zhouming Deng et al. This is an open access article distributed under the Creative Commons Attribution License, which permits unrestricted use, distribution, and reproduction in any medium, provided the original work is properly cited.

\begin{abstract}
This study aimed to examine the expression of and the relationship between CD44V6, CDH11, and $\beta$-catenin. The expression of these cell adhesion molecules was detected in 90 osteosarcoma and 20 osteochondroma specimens using immunohistochemistry. Associations between these parameters and clinicopathological data were also examined. The expression rates of CD44V6, CDH11, and $\beta$-catenin were $25.0 \%(5 / 20), 70.0 \%(14 / 20)$, and $20.0 \%(4 / 20)$ in osteochondroma specimens, respectively. Compared to osteochondromas, the proportions of expression of CD44V6 and $\beta$-catenin in osteosarcoma specimens increased to $65.6 \%$ (59/90) and $60.0 \%$ (54/90), respectively. However, the expression rate of CDH11 in osteosarcomas was reduced to 40.0\% (36/90). The expression of these markers was significantly associated with metastasis and overall survival $(P<0.05)$. Survival analysis revealed that patients with increased expression of CD44V6 and $\beta$-catenin as well as decreased expression of CDH11 were correlated with a shorter survival time. Multivariate analysis indicated that clinical stage, metastasis status, and the expression of CD44V6, CDH11, and $\beta$-catenin were found to be associated with overall survival. Further, the expression of $\beta$-catenin and that of CD44V6 were positively correlated with each other. Thus, our results indicated abnormal expression of CD44V6, CDH11, and $\beta$-catenin in osteosarcomas and osteochondromas, which may provide important indicators for further research.
\end{abstract}

\section{Introduction}

Primary malignant tumors of bone are extremely rare neoplasm accounting for less than $0.2 \%$ of all cancers, although the true incidence is not easy to determine owing to the rarity of these tumors $[1,2]$. Osteosarcoma is the most common form of primary bone cancer and accounts for approximately $19 \%$ of all malignant tumors of the bone. It is the third most common malignant tumor in teenagers [3]. Current treatments for osteosarcoma include surgical resection of both primary and pulmonary lesions, chemotherapy, and radiotherapy. Disease-free survival escalated from $<20 \%$ prior to the introduction of effective chemotherapy to around $60 \%$ and overall survival to $60-70 \%[2,4,5]$. However, the prognoses were unsatisfactory in patients with undetectable metastases at diagnosis [5]. In fact, only approximately $20-25 \%$ of newly diagnosed patients have clinically detectable metastatic disease at diagnosis [6]. At present, the ability to predict the prognosis of osteosarcomas is limited. Therefore, identifying prognostic markers of survival in osteosarcomas could be informative for selecting proper management. Traditional prognostic markers, such as gender, age, tumor location, disease-free interval, tumor doubling time, representation, and number of detectable pulmonary metastases, have had limited success in identifying those patients that need aggressive chemotherapy and those that do not [7]. More recently, the expression of certain biological molecules has been identified as potential prognostic markers for osteosarcoma metastasis, including the expression of CD44V6, CDH11, and $\beta$-catenin [7-9].

CD44 is a transmembrane glycoprotein, and some variant isoforms of CD44 (CD44V) are reportedly associated with increased invasion, metastasis, and poor prognosis [10-12], particularly the CD44 variant isoform V6 (CD44V6) [1113]. It has been reported that CD44V6 can regulate the extracellular matrix, promote cell motility, and suppress 
tumor apoptosis. In fact, CD44V6 has been implicated in promoting tumor progression [11].

Cadherins comprise a major class of cell-cell adhesion molecules that interact with catenins to regulate adhesion and migration by $\mathrm{Ca}^{2+}$-dependent hemophilic interactions $[14,15]$. These interactions are involved in maintenance of the tissue structure and morphogenesis as well as limiting cell movement and proliferation, thus resulting in tumor inhibition [16, 17]. Cadherin-11 (CDH11), also known as osteoblast cadherin, has been assigned a wide range of roles in relation to its influence on cancer cell behavior. It has been indicated that CDH11 expression may be useful as a prognostic marker of disease progression and survival in osteosarcomas [7].

The Wnt/ $\beta$-catenin pathway has been implicated in the progression and pathogenesis of many forms of human cancer [18]. However, it has yet to be clarified whether Wnt $/ \beta$ catenin signaling plays a role in osteosarcoma development. Several studies have reported that both nuclear and cytoplasmic $\beta$-catenin could be detected in osteosarcomas [19] or correlated with metastasis [9].

CD44V6, CDH11, and $\beta$-catenin belong to the family of cell adhesion molecules (CAM), which have been implicated in all aspects of cell growth, migration, and differentiation in vertebrate cells. Much research is focused on the relationship between CAM and cancer [7, 9, 11, 13-15, 19]. As part of ongoing efforts to understand the roles of and interactions between CD44V6, CDH11, and $\beta$-catenin in osteosarcomas, we report in this study our findings regarding the immunohistochemical expression and clinicopathological features of these biomarkers in human osteosarcomas. We have also analyzed the correlation of CD44V6, CDH11, and $\beta$-catenin with each other.

\section{Materials and Methods}

2.1. Patients and Samples. All patients or their guardians provided informed consent for participation in this study. This study was approved by the local research ethics committee (Zhongnan Hospital of Wuhan University). The exclusion criterion was inadequate follow-up data as well as preoperative chemotherapy or radiotherapy. Surgically resected osteosarcoma specimens were collected from 90 patients at Zhongnan Hospital of Wuhan University and Hubei Cancer Hospital, Hubei, China, between January 2000 and March 2008. The specimens were obtained from the primary site from 50 patients with metastases and 40 without metastases. No diagnostic biopsies were selected for this study. A total of 20 osteochondroma specimens were used as controls. Formalin-fixed, paraffin-embedded blocks were retrieved from the Department of Pathology. All the samples were evaluated for diagnosis by 3 experienced pathologists. Of the 90 patients, 53 were male and 37 were female. The median age of the patients was 18.3 years, with a range of 8-58 years. Clinical stage was graded according to the standard of Enneking et al. [20]; there were two cases of stage IB, 24 cases of stage IIA, 39 cases of stage IIB, and 25 cases of stage III. For the 20 osteochondroma patients, 12 were male and 8 were female, with an average age of 60.4 years ranging from 21 to 78 years.

2.2. Immunohistochemistry. The tissues were fixed in $10 \%$ buffered formalin and processed using standard procedures. The sections $(4-5 \mu \mathrm{m})$ obtained from representative tissue sample blocks were heated at $60^{\circ} \mathrm{C}$ for $20 \mathrm{~min}$, deparaffinized with xylene, and rehydrated using graded ethanol. After quenching endogenous peroxidase activity with $3 \%$ hydrogen peroxide in methanol for $10 \mathrm{~min}$, the slides were microwaved in $10 \mathrm{mmol} / \mathrm{L}$ sodium citrate $(\mathrm{pH} 6.0)$ for $15 \mathrm{~min}$ to retrieve the antigen. The elimination of nonspecific staining was performed with diluted normal goat serum. The sections were then incubated at $37^{\circ} \mathrm{C}$ for $1 \mathrm{~h}$ with primary antibodies against CD44V6 (ZSGB-BIO, China), CDH11 (RD Biosciences, USA), and $\beta$-catenin (ZSGB-BIO, China). Following three successive rinses with phosphate buffered saline (PBS), they were further incubated with secondary antibody (Maixin Bio, China) for $40 \mathrm{~min}$ at $37^{\circ} \mathrm{C}$. The sections were allowed to develop using a DAB color kit (ZSGB-BIO, China) for $5 \mathrm{~min}$ and then were counterstained with hematoxylin. For each protein, the immunostaining in patient samples was compared with osteochondroma as a normal control, while negative controls were obtained by omitting the primary antibody, substituted by PBS.

2.3. Evaluation of Immunohistochemistry. The "immunohistochemical score" (IHS) was calculated based on previously published research [21]. Immunoreactivity was evaluated prior to the collection of the patient identity and clinical information. The IHC classification of positivity was scored as follows: (1) $\leq 25 \%$ of cells staining positively; (2) $26 \%-$ $50 \%$ of cells staining positively; (3) $51 \%-75 \%$ of cells staining positively; (4) $76 \%-100 \%$ of cells staining positively. The intensity of the immunoexpression was rated as negative (0), weak (1), moderate (2), or strong (3). A consensus was achieved by three of the authors in all cases. The final IHS was obtained by multiplying the score of extent and intensity. The IHS of each specimen was categorized into four groups: ,$-(0-2) ;+,(3-5) ;++,(6-8) ;+++,(9-12)$. Scores of $0-5$ were designated as low expression, while scores of 6-12 were designated as expression.

2.4. Statistical Analysis. The whole statistical analysis was performed with SPSS version 18.0 software (SPSS Inc, Chicago, USA). The correlation between antigen expression and the clinicopathological parameters was assessed by chisquare test or Kruskal-Wallis test when chi-square test was not suitable. Survival rates were estimated by Kaplan-Meier statistics and survival curves were compared by using the Log-rank test. A Cox regression method was used for multivariate analysis. In this paper, we only investigated the relation between expressions of the three markers with overall survival; the metastasis-free survival was not studied. Survival was calculated from the time of the primary operation. An observation was censored at the last followup if the patient was alive or had died of a cause other than osteosarcoma. The correlation between CD44V6, CDH11, and $\beta$-catenin was 
TABLE 1: Expression of CD44V6, CDH11, and $\beta$-catenin in osteosarcomas and osteochondromas.

\begin{tabular}{|c|c|c|c|c|c|c|c|c|c|c|}
\hline & \multirow[b]{2}{*}{ Cases } & \multicolumn{3}{|c|}{ CD44V6 expression } & \multicolumn{3}{|c|}{ CDH11 expression } & \multicolumn{3}{|c|}{$\beta$-catenin expression } \\
\hline & & Expression & $\begin{array}{c}\text { Low } \\
\text { expression }\end{array}$ & $P$ & Expression & $\begin{array}{c}\text { Low } \\
\text { expression }\end{array}$ & $P$ & Expression & $\begin{array}{c}\text { Low } \\
\text { expression }\end{array}$ & $P$ \\
\hline OS & 90 & $\begin{array}{c}59 \\
(65.6 \%)\end{array}$ & $\begin{array}{c}31 \\
(34.4 \%)\end{array}$ & 0.0009 & $\begin{array}{c}36 \\
(40.0 \%)\end{array}$ & $\begin{array}{c}54 \\
(60.0 \%)\end{array}$ & 0.042 & $\begin{array}{c}54 \\
(60.0 \%)\end{array}$ & $\begin{array}{c}36 \\
(40.0 \%)\end{array}$ & 0.001 \\
\hline $\mathrm{OC}$ & 20 & $\begin{array}{c}5 \\
(25.0 \%)\end{array}$ & $\begin{array}{c}15 \\
(75.0 \%)\end{array}$ & & $\begin{array}{c}14 \\
(70.0 \%)\end{array}$ & $\begin{array}{c}6 \\
(30.0 \%)\end{array}$ & & $\begin{array}{c}4 \\
(20.0 \%)\end{array}$ & $\begin{array}{c}16 \\
(80.0 \%)\end{array}$ & \\
\hline
\end{tabular}

OS: osteosarcoma, OC: osteochondroma.

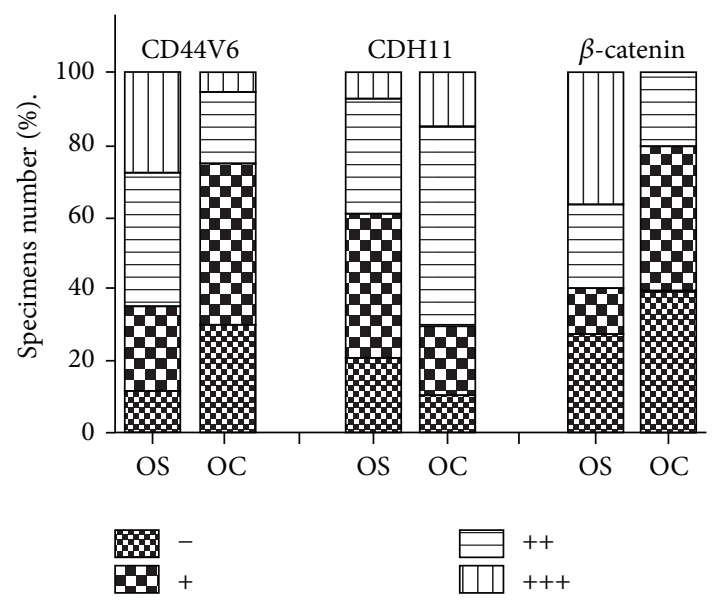

Figure 1: The immunohistochemical expression of CD44V6, $\mathrm{CDH} 11$, and $\beta$-catenin in osteosarcoma and osteochondroma samples.

assessed by Spearman's correlation analysis. A $P$ value of less than 0.05 was considered statistically significant for all tests.

\section{Results}

3.1. Expression of CD44V6, CDH11, and $\beta$-Catenin and Their Correlations with Clinicopathological Features of Patients with Osteosarcoma. The different expression levels of the three markers in osteosarcoma and osteochondroma are depicted in Figure 1 (Table 1). Of the osteochondroma cases, diffuse expression of CD44V6 was observed in 5 cases $(25.0 \%)$, CDH11 in 14 cases $(70.0 \%)$, and $\beta$-catenin in 4 cases $(20.0 \%)$ (Table 1$)$. In contrast, the proportions of expression of CD44V6 and $\beta$-catenin in osteosarcoma cases were increased to $65.6 \%(59 / 90)$ and $60.0 \%(54 / 90)$, respectively. However, the rate of expression of CDH11 in osteosarcomas was reduced compared to osteochondromas to $40.0 \%$ $(36 / 90)$. Representative staining in osteosarcoma specimens is depicted in Figure 2. The chi-square test demonstrated that the difference in expression of these three parameters between osteosarcomas and osteochondromas demonstrated statistical significance $(P<0.05$, Table 1$)$.

Total CD44V6, CDH11, and $\beta$-catenin protein staining was associated with metastasis $(P<0.05)$. The expression of CD44V6, CDH11, and $\beta$-catenin was not significantly associated with age, gender, tumor site, and histological subtype (Table 2).
3.2. Prognostic Values of CD44V6, CDH11 and $\beta$-Catenin Expressions. Survival analysis was conducted using KaplanMeier curves for overall survival and univariate analysis. All three potential surrogate endpoints proved to alter the survival probability with $P$ values below 0.01 (Log-rank test, Figure 3). Based on the expression of all three proteins, the patients were reassigned to eight groups. The survival analysis revealed that a shorter survival time was correlated with patients who demonstrated expression of CD44V6 and $\beta$ catenin as well as low expression of CDH11. On the other hand, low expression of CD44V6 and $\beta$-catenin as well as expression of CDH11 indicated better survival $(P<0.05$, Figure 4).

Proceeding to multivariate analysis, Cox multivariate analysis showed that the expression of the three markers, clinical stage, and metastasis status remained significantly associated with overall survival, whereas age and gender were not (Table 3).

3.3. The Correlation of CD44V6, CDH11, and $\beta$-Catenin in Osteosarcoma. To determine whether these CAMs were associated with each other in osteosarcomas, we performed correlative analysis. The results revealed that CD44V6 expression was positively correlated with the expression of $\beta$ catenin $(r=0.768, P<0.001$, Table 4$)$.

\section{Discussion}

Osteosarcoma is the most frequent primary cancer of bone (incidence: $0.2-0.3 / 100000 /$ year). The incidence is higher in adolescents (0.8-1.1/100 000/year at age 15-19), where it accounts for $>10 \%$ of all solid cancers [2]. Currently, there is a lack of understanding of the molecular mechanisms leading to the development and progression of osteosarcomas. Regardless of intensifying and modifying chemotherapy, limited improvements to survival of osteosarcoma patients have been achieved over the past 20 years [22]. The development of metastasis to the lungs represents the most common cause of death in osteosarcoma patients. Despite growing evidence implicating roles for particular molecular markers and pathways in the initiation and progression of osteosarcoma, their clinical significance remains debatable. Recently, evidence has revealed that a phenomenon called "chromothripsis" can trigger between tens and hundreds of genomic rearrangements in multiple cancer samples, promoting the development of cancer. The complex genomic rearrangement, with frequent copy number changes, confined to localized 


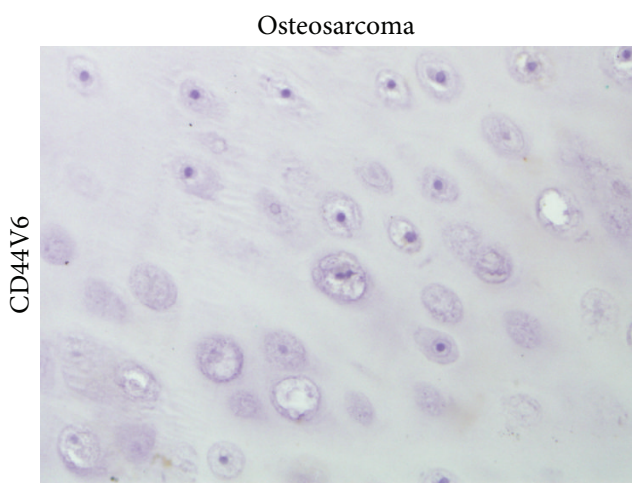

(a)

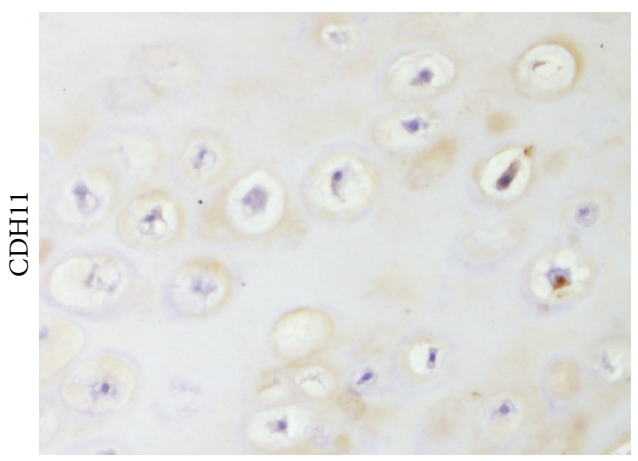

(c)

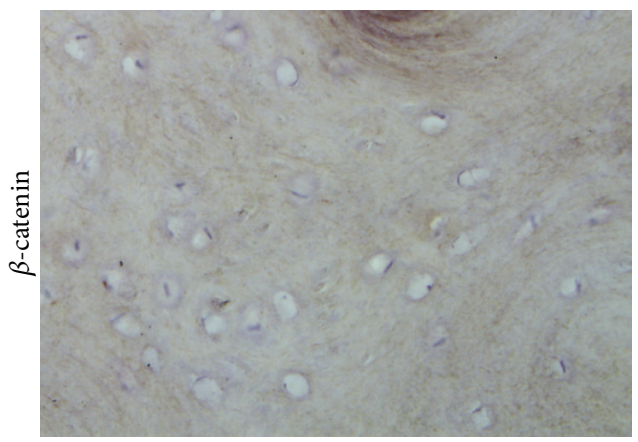

(e)

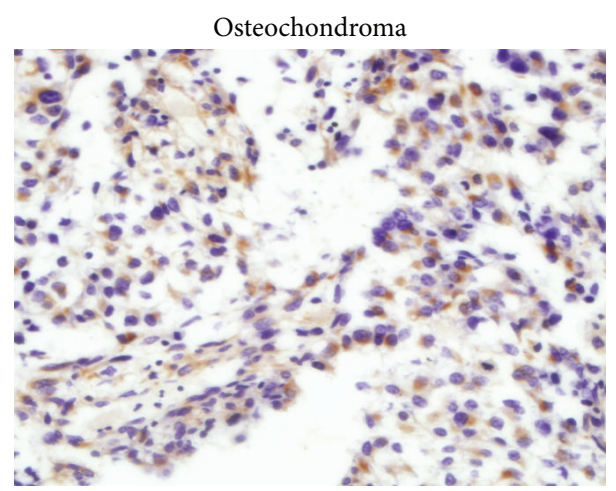

(b)

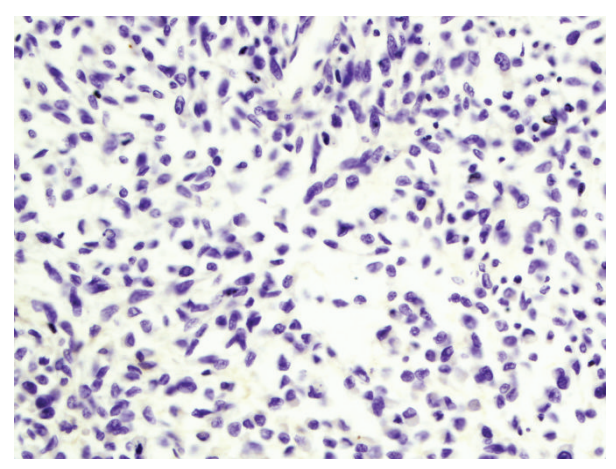

(d)

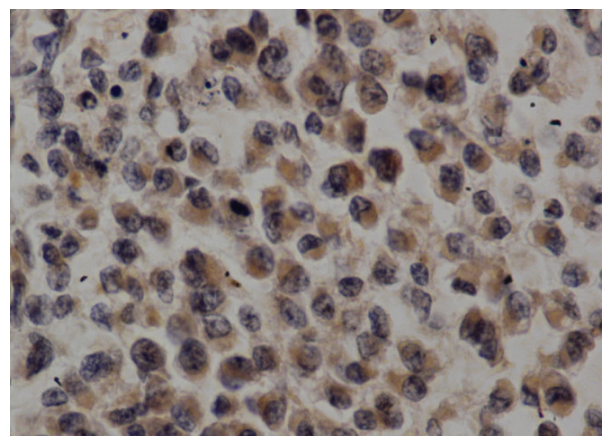

(f)

FIgURE 2: Representative expression of CD44V6, CDH11, and $\beta$-catenin in osteosarcoma and osteochondroma samples.

genomic regions rapidly alternating between usually no more than three different states, occurred in at least 2-3\% of all cancers. The genomic features imply chromosome breaks occur in a single catastrophic event rather than as a cumulative acquisition of mutations. The "chromothripsis" was especially common in osteosarcomas (more than 30\%), which was suggested as a critical event in the conversion of a normal cell to a cancerous cell [23]. Another study revealed a notable association between TP53 mutation and "chromothripsis" [24]. The metastatic cascade remains a complex process, and the CAMs play an important role in the first step of tumor metastasis $[25,26]$. To confirm our hypothesis that CD44V6, CDH11, and $\beta$-catenin are potential tumor markers, their protein level and relationship with prognosis were analyzed using immunochemistry.

Our results revealed that CD44V6 was expressed in $25.0 \%$ of the collected samples of osteochondroma, whereas,
CD44V6 was expressed in $65.6 \%$ of osteosarcoma samples. The findings in our study are consistent with previous research demonstrating that the expression of CD44V6 was correlated with metastasis and poor prognosis in patients with osteosarcoma [8]. It was also reported that the higher expression of CD44V6 correlated with metastasis and poorer survival in patients with other tumors (i.e., pancreatic cancer, colorectal cancer, ovarian cancer, bladder cancer, lung cancer, and glioblastoma multiforme) [27-32]. In addition, Fan et al. [33] performed a meta-analysis, in which CD44V6 positive cells $(\mathrm{OR}=0.36, P=0.02)$ were significantly associated with poor overall survival in patients with colorectal cancer. The mechanism of CD44V6 promoting the metastasis of cancer may be attributed to its interactions with various components of the extracellular matrix and its involvement in cell adhesion and critical signaling pathways, for example, Ras and Akt [34, 35]. Nakajima et al. [36] suggested that 


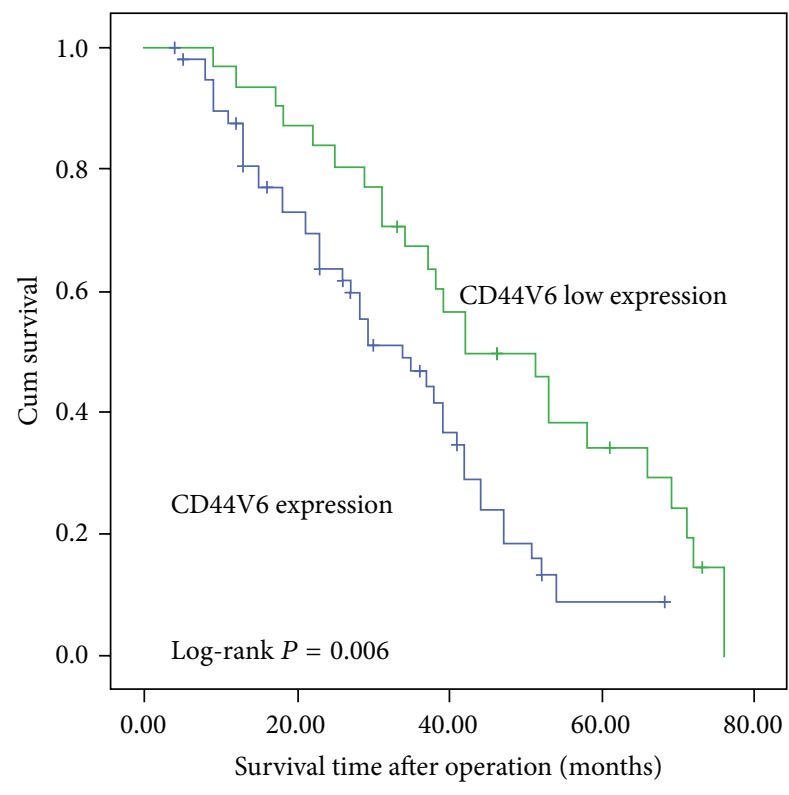

(a)

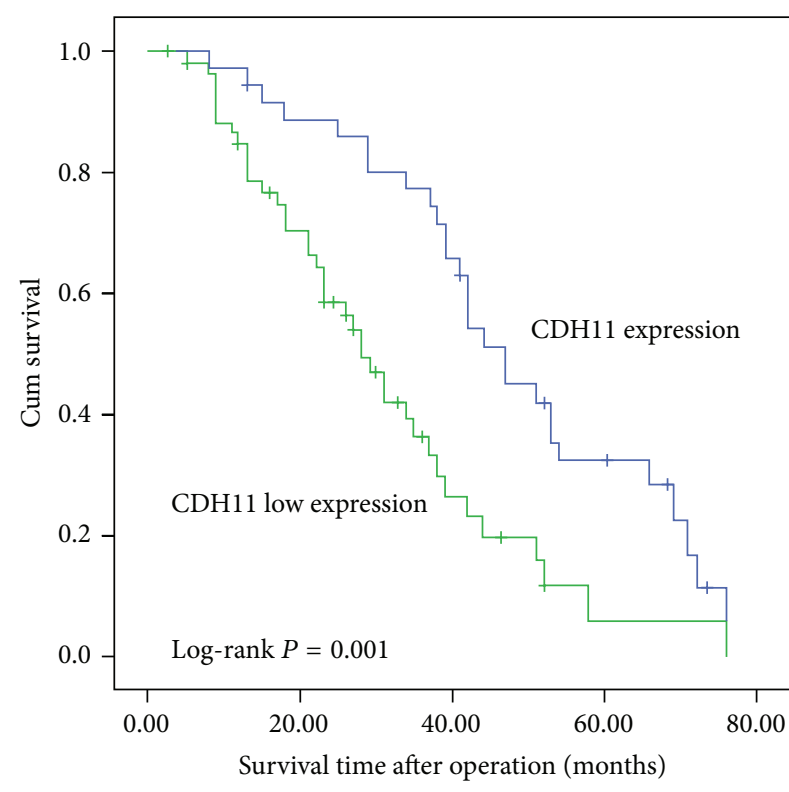

(b)

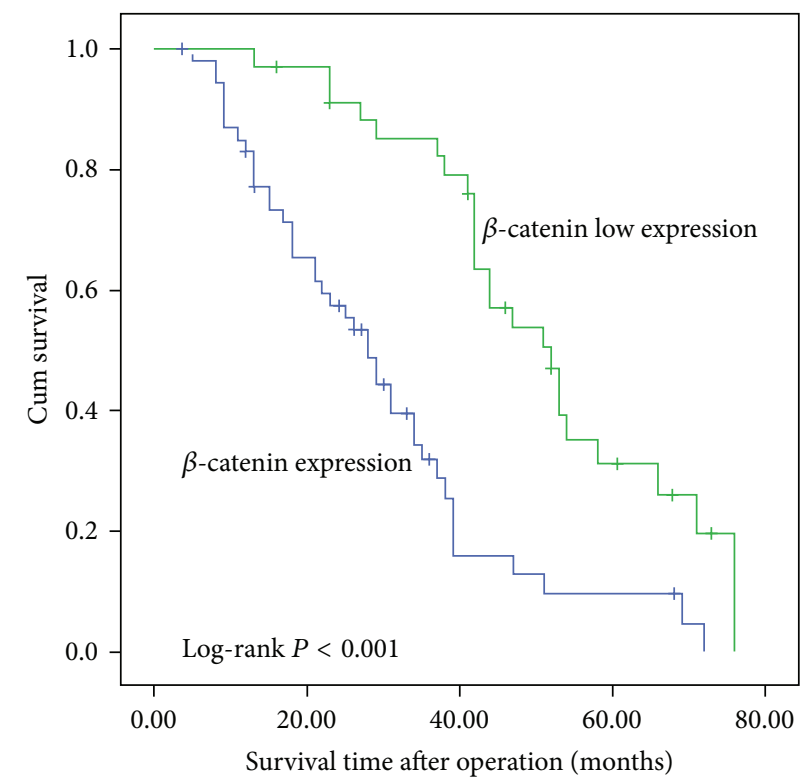

(c)

FIgURE 3: Postoperative survival curves for patients, according to the expression of CD44V6, CDH11, and $\beta$-catenin, respectively.

CD44V6 could be an oncofetal protein in the bone tissue, which could be expressed in the osteosarcoma when it metastasizes. Furthermore, phase I clinical trials of CD44V6 antibodies that were either radiolabeled or covalently linked to a toxin were investigated in patients affected by head and neck squamous cell carcinomas, with the outcome of the trial providing promising results [37]. These results reinforce our findings. However, there are other inconsistent reports of the implications of CD44V6 expression in cancer. Yang et al. [38] found that decreased CD44V6 expression promoted the recurrence and carcinogenesis of parotid pleomorphic adenoma. Spafford et al. [39] reported that increased CD44V6 expression was consistent with longer survival $(P<0.02)$ of patients with laryngeal squamous cell carcinoma. In addition, the association of CD44V6 expression with malignancy and survival could not be confirmed in several studies investigating osteosarcomas as well as other tumors [40-43]. Therefore, the suitability of CD44V6 expression to be used as a prognostic marker remains a matter of debate.

In this study, a significant correlation was found between CDH11 expression and patient survival, which is consistent with a previous study [7]. Several studies have suggested that CDH11 displays tumor suppressor properties in osteosarcomas and other tumors [44-48]. The loss or decrease of CDH11 expression plays an important role in osteosarcoma metastasis [47]. Kashima et al. [48] have found that 
TABLE 2: Correlation between the expression of CD44V6, CDH11, and $\beta$-catenin and clinicopathological data.

\begin{tabular}{|c|c|c|c|c|c|c|c|c|c|}
\hline \multirow{2}{*}{ Clinical features } & \multicolumn{2}{|c|}{ CD44V6 expression } & \multirow[b]{2}{*}{$P$} & \multicolumn{2}{|c|}{ CDH11 expression } & \multicolumn{4}{|c|}{$\beta$-catenin expression } \\
\hline & Expression & Low expression & & Expression & Low expression & $P$ & Expression & Low expression & $P$ \\
\hline Age & & & 0.168 & & & 0.418 & & & 0.928 \\
\hline$\leq 50$ & 18 & 14 & & 11 & 21 & & 19 & 13 & \\
\hline$>50$ & 41 & 17 & & 25 & 33 & & 35 & 23 & \\
\hline Gender & & & 0.432 & & & 0.600 & & & 0.097 \\
\hline Male & 33 & 20 & & 20 & 33 & & 28 & 25 & \\
\hline Female & 26 & 11 & & 16 & 21 & & 26 & 11 & \\
\hline Histological classification & & & 0.179 & & & 0.382 & & & 0.891 \\
\hline Osteoblastic & 32 & 11 & & 18 & 25 & & 27 & 16 & \\
\hline Chondroblastic & 11 & 7 & & 9 & 9 & & 11 & 7 & \\
\hline Fibroblastic & 8 & 5 & & 2 & 11 & & 8 & 5 & \\
\hline Telangiectatic & 4 & 7 & & 5 & 6 & & 5 & 6 & \\
\hline Mixed & 4 & 1 & & 2 & 3 & & 3 & 2 & \\
\hline Primary site & & & 0.495 & & & 0.667 & & & 0.367 \\
\hline Femur & 28 & 15 & & 17 & 26 & & 23 & 20 & \\
\hline Tibia & 12 & 9 & & 11 & 10 & & 16 & 5 & \\
\hline Humerus & 8 & 5 & & 4 & 9 & & 8 & 5 & \\
\hline Fibula & 1 & 1 & & 1 & 1 & & 2 & 0 & \\
\hline Ilium & 5 & 1 & & 1 & 5 & & 3 & 3 & \\
\hline Other & 5 & 0 & & 2 & 3 & & 2 & 3 & \\
\hline Metastasis & & & $0.02^{*}$ & & & $0.009^{*}$ & & & $0.002^{*}$ \\
\hline Yes & 38 & 12 & & 14 & 36 & & 37 & 13 & \\
\hline No & 21 & 19 & & 22 & 18 & & 17 & 23 & \\
\hline
\end{tabular}

TABLE 3: Multivariate survival analysis of overall survival in patients with osteosarcoma.

\begin{tabular}{lccc}
\hline Variables & $P$ & RR & 95\% CI for RR \\
\hline Age & 0.321 & 1.019 & $0.982-1.057$ \\
Gender & 0.221 & 0.749 & $0.471-1.190$ \\
Metastasis status & 0.019 & 2.067 & $1.129-3.784$ \\
Clinical stage & 0.000 & 2.288 & $1.559-3.360$ \\
CD44V6 expression & 0.042 & 0.586 & $0.350-0.982$ \\
CDH11 expression & 0.010 & 1.934 & $1.172-3.191$ \\
$\beta$-catenin expression & 0.002 & 0.425 & $0.249-0.727$ \\
\hline
\end{tabular}

TABLE 4: The correlation analysis between expression of CD44V6, CDH11, and $\beta$-catenin.

\begin{tabular}{lccc}
\hline & CD44V6 & CDH11 & $\beta$-catenin \\
\hline CD44V6 & & $r s=0.200$, & $r s=0.768$, \\
& & $P=0.059$ & $P<0.001^{*}$ \\
CDH11 & $r s=0.200$, & & $r s=-0.231$, \\
& $P=0.059$ & & $P=0.029^{*}$ \\
$\beta$-catenin & $r s=0.768$, & $r s=-0.231$, & \\
& $P<0.001^{*}$ & $P=0.029^{*}$ & \\
\hline
\end{tabular}

rs: Spearman's rank correlation coefficient, two-tailed significances, ${ }^{*} P<$ 0.05 .

osteosarcoma metastasis can be prevented by restoration of CDH11 expression using an in vivo metastasis assay.
It has been suggested that tumor-promoting inflammation and antitumor immunity coexist at different points along the path of tumor progression [49], and a recent report has demonstrated that CDH11 was a key mediator of fibroblast inflammation [50]. Consequently, it appears that CDH11 may be involved in osteosarcoma invasion and metastasis through a potential link between inflammation and tumor development.

$\beta$-catenin is an intracellular protein with two important cellular functions: The cadherin-bound $\beta$-catenin is required for cell adhesion, while nuclear $\beta$-catenin transmits extracellular-initiated Wnt signals to the nucleus. $\beta$-catenin is involved with differentiation and proliferation of cells in a wide variety of tissues, and the Wnt signaling pathway has emerged as an essential pathway in skeletal development and disease [51]. Several studies have indicated that active Wnt signaling is conducive to osteosarcoma progression based on cytoplasmic or membranous $\beta$-catenin staining $[9,19,52]$. However, this may not prove the role of active Wnt signaling owing to the dual role of $\beta$-catenin. Considering this pivotal role of $\beta$-catenin, we also analyzed $\beta$-catenin expression in our study. Cytoplasmic immunostaining was observed in most cases of osteosarcoma (74/90), and we observed that the expression of $\beta$-catenin was significantly increased in osteosarcomas compared to osteochondromas. Furthermore, low expression of $\beta$-catenin was correlated with longer survival time. Several studies have revealed that positive cytoplasmic $\beta$-catenin expression was associated with the 


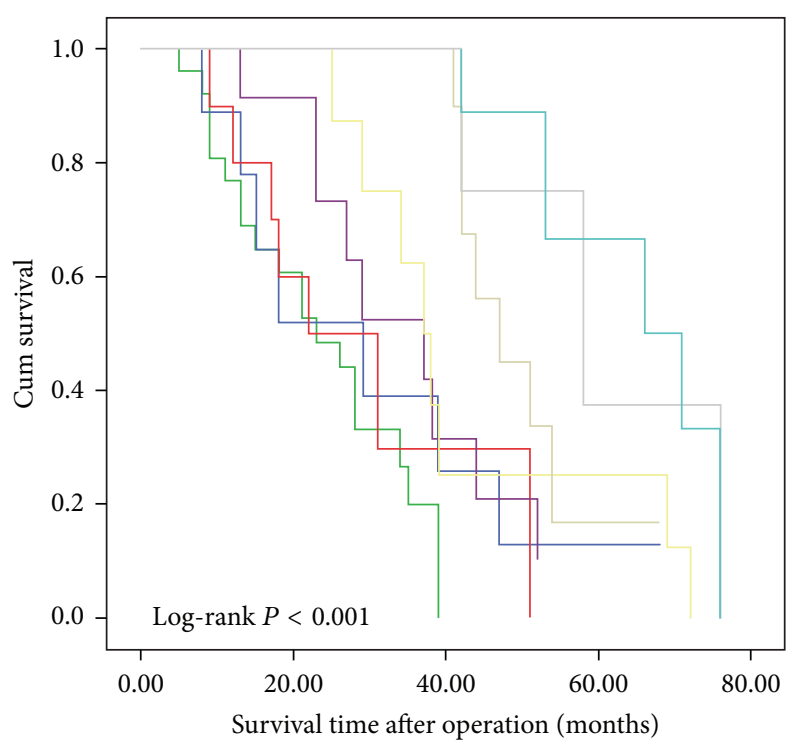

$\neg \mathrm{CD} 44 \mathrm{~V} 6, \mathrm{CDH} 11, \beta$-catenin expression

$\neg \mathrm{CD} 44 \mathrm{~V} 6, \beta$-catenin expression; $\mathrm{CDH} 11$ low expression CD44V6, CDH11 expression; $\beta$-catenin low expression

$\neg$ CD44V6 expression; CDH11, $\beta$-catenin low expression CD44V6 low expression; CDH11, $\beta$-catenin expression

$\neg \mathrm{CD} 44 \mathrm{~V} 6, \mathrm{CDH} 11$ low expression; $\beta$-catenin expression

$\neg \mathrm{CD} 44 \mathrm{~V} 6, \beta$-catenin low expression; $\mathrm{CDH} 11$ expression

CD44V6, CDH11, $\beta$-catenin low expression

Figure 4: Postoperative survival curves for patients, according to the conjoint analysis of CD44V6, CDH11, and $\beta$-catenin expression.

development of metastasis both in vivo and in vitro $[9,52-$ 54]. Although the deregulation of $\beta$-catenin is thought to play an important role in oncogenesis of osteosarcomas, the role of Wnt signaling in osteosarcomas remains controversial $[18,22,53,55,56]$. Recently, a study has indicated that $\beta$-catenin may interact with other proteins, such as NFkappa B, during oncogenesis [57]. Further studies using new immunohistochemical markers of the Wnt signaling pathway are needed to investigate the potential role of this pathway in osteosarcoma pathogenesis.

We also found that the expression of CD44V6 was significantly positively correlated with that of $\beta$-catenin $(P<$ $0.001)$. The correlation between CD $44 \mathrm{~V} 6$ and $\beta$-catenin was concordant with a previous study, which showed that CD44 overexpression (CD44S and CD44V6) was associated with activation of $\beta$-catenin, suggesting CD44 is one of the target genes of $\beta$-catenin [58].

An obvious shortcoming of our study is that immunohistochemistry is, at best, a semiquantitative technique. The results should be considered exploratory and caution should be taken in interpreting data. Moreover, as for other prognostic factors, the functions of these molecular markers may vary depending on the tissue context. These results may not be applicable to other tumor types. Another limitation of our study is that osteochondromas were used as a control due to limited conditions, and therefore the results may not be as convincing as a comparison with normal bone specimens.
Further studies using normal bone tissue as controls are needed. Despite these limitations, noteworthy results were acquired. The expression of CD44V6, CDH11, and $\beta$-catenin could be a potential prognostic indicator, especially conjoint analysis of the three markers. The relevant molecular mechanisms require further investigation. Considering that osteosarcoma is a very rare disease, this emphasizes the need for multi-institutional collaboration to identify and validate new biomarkers.

\section{Conflict of Interests}

The authors declare that they have no competing interests.

\section{Acknowledgment}

This study was supported by the Fundamental Research Funds for the Central Universities (no. 2012303020207).

\section{References}

[1] National Comprehensive Cancer Network, NCCN Clinical Practice Guidelines in Oncology-Bone Cancer, 2012, http://www .nccn.org/professionals/physician_gls/f_guidelines.asp.

[2] The ESMO/European Sarcoma Network Working Group, "Bone sarcomas: ESMO Clinical Practice Guidelines for diagnosis, treatment and follow-up," Annals of Oncology, vol. 23, supplement 7, pp. viil00-vii109, 2012.

[3] C. Kong and M. F. Hansen, "Biomarkers in osteosarcoma," Expert Opinion on Medical Diagnostics, vol. 3, no. 1, pp. 13-23, 2009.

[4] E. R. Wagner, G. Luther, G. Zhu et al., "Defective osteogenic differentiation in the development of osteosarcoma," Sarcoma, vol. 2011, Article ID 325238, 12 pages, 2011.

[5] T. S. Osborne and C. Khanna, "A review of the association between osteosarcoma metastasis and protein translation," Journal of Comparative Pathology, vol. 146, no. 2-3, pp. 132-142, 2012.

[6] C. M. Hattinger, M. Pasello, S. Ferrari, P. Picci, and M. Serra, "Emerging drugs for high-grade osteosarcoma," Expert Opinion on Emerging Drugs, vol. 15, no. 4, pp. 615-634, 2010.

[7] G. Nakajima, A. Patino-Garcia, S. Bruheim et al., "CDH11 expression is associated with survival in patients with osteosarcoma," Cancer Genomics and Proteomics, vol. 5, no. 1, pp. 37-42, 2008.

[8] M. Kuryu, T. Ozaki, K. Nishida, M. Shibahara, A. Kawai, and H. Inoue, "Expression of CD44 variants in osteosarcoma," Journal of Cancer Research and Clinical Oncology, vol. 125, no. 11, pp. 646-652, 1999.

[9] K. Iwaya, H. Ogawa, M. Kuroda, M. Izumi, T. Ishida, and K. Mukai, "Cytoplasmic and/or nuclear staining of beta-catenin is associated with lung metastasis," Clinical and Experimental Metastasis, vol. 20, no. 6, pp. 525-529, 2003.

[10] R. L. Brown, L. M. Reinke, M. S. Damerow et al., "CD44 splice isoform switching in human and mouse epithelium is essential for epithelial-mesenchymal transition and breast cancer progression," Journal of Clinical Investigation, vol. 121, no. 3, pp. 1064-1074, 2011.

[11] T. Jung, W. Gross, and M. Zöller, "CD44v6 coordinates tumor matrix-triggered motility and apoptosis resistance," Journal of Biological Chemistry, vol. 286, no. 18, pp. 15862-15874, 2011. 
[12] X. G. Zhang, X. F. Lu, X. M. Jiao, B. Chen, and J. X. Wu, "PLK1 gene suppresses cell invasion of undifferentiated thyroid carcinoma through the inhibition of CD44v6, MMP-2 and MMP-9," Experimental and Therapeutic Medicine, vol. 4, no. 6, pp. 1005-1009, 2012.

[13] Z. J. Zhou, Z. Dai, S. L. Zhou et al., “Overexpression of HnRNP A1 promotes tumor invasion through regulating CD44v6 and indicates poor prognosis for hepatocellular carcinoma," International Journal of Cancer, vol. 132, no. 5, pp. 1080-1089, 2013.

[14] H. Kaur, P. J. Phillips-Mason, S. M. Burden-Gulley et al., "Cadherin-11, a marker of the mesenchymal phenotype, regulates glioblastoma cell migration and survival in vivo," Molecular Cancer Research, vol. 10, no. 3, pp. 293-304, 2012.

[15] T. Yagi and M. Takeichi, "Cadherin superfamily genes: functions, genomic organization, and neurologic diversity," Genes and Development, vol. 14, no. 10, pp. 1169-1180, 2000.

[16] G. Berx and F. van Roy, "Involvement of members of the cadherin superfamily in cancer," Cold Spring Harbor Perspectives in Biology, vol. 1, no. 6, Article ID a003129, 2009.

[17] J. Brieger, A. Duesterhoeft, C. Brochhausen, J. Gosepath, C. J. Kirkpatrick, and W. J. Mann, "Recurrence of pleomorphic adenoma of the parotid gland-predictive value of cadherin-11 and fascin," APMIS, vol. 116, no. 12, pp. 1050-1057, 2008.

[18] D. M. Thomas, "Wnts, bone and cancer," Journal of Pathology, vol. 220, no. 1, pp. 1-4, 2010.

[19] R. C. Haydon, A. Deyrup, A. Ishikawa et al., "Cytoplasmic and/ or nuclear accumulation of the $\beta$-catenin protein is a frequent event in human osteosarcoma," International Journal of Cancer, vol. 102, no. 4, pp. 338-342, 2002.

[20] W. F. Enneking, S. S. Spanier, and M. Goodman, "A system for the surgical staging of musculoskeletal sarcoma," Clinical Orthopaedics and Related Research, vol. 153, pp. 106-120, 1980.

[21] B. D. Gun, B. Bahadir, S. Bektas et al., "Clinicopathological significance of fascin and CD44v6 expression in endometrioid carcinoma," Diagnostic Pathology, vol. 7, article 80, 2012.

[22] Y. Cai, A. B. Mohseny, M. Karperien, P. C. W. Hogendoorn, G. Zhou, and A.-M. Cleton-Jansen, "Inactive Wnt/ $\beta$-catenin pathway in conventional high-grade osteosarcoma," Journal of Pathology, vol. 220, no. 1, pp. 24-33, 2010.

[23] P. J. Stephens, C. D. Greenman, B. Fu et al., "Massive genomic rearrangement acquired in a single catastrophic event during cancer development," Cell, vol. 144, no. 1, pp. 27-40, 2011.

[24] T. Rausch, D. T. W. Jones, M. Zapatka et al., "Genome sequencing of pediatric medulloblastoma links catastrophic DNA rearrangements with TP53 mutations," Cell, vol. 148, no. 1-2, pp. 5971, 2012.

[25] S. Saadatmand, E. M. de Kruijf, A. Sajet et al., "Expression of cell adhesion molecules and prognosis in breast cancer," The British Journal of Surgery, vol. 100, no. 2, pp. 252-260, 2013.

[26] J. S. Park, K. M. Kim, M. H. Kim et al., "Resveratrol inhibits tumor cell adhesion to endothelial cells by blocking ICAM1 expression," Anticancer Research, vol. 29, no. 1, pp. 355-362, 2009.

[27] G. Zhou, D. Chiu, D. Qin et al., "Detection and clinical significance of CD44v6 and integrin-betal in pancreatic cancer patients using a triplex real-time RT-PCR assay," Applied Biochemistry and Biotechnology, vol. 167, no. 8, pp. 2257-2268, 2012.

[28] A. Garouniatis, A. Zizi-Sermpetzoglou, S. Rizos, A. Kostakis, N. Nikiteas, and A. G. Papavassiliou, "FAK, CD44v6, c-Met and EGFR in colorectal cancer parameters: tumour progression, metastasis, patient survival and receptor crosstalk," International Journal of Colorectal Disease, vol. 28, no. 1, pp. 9-18, 2013.
[29] J. Shi, Z. Zhou, W. Di, and N. Li, "Correlation of CD44v6 expression with ovarian cancer progression and recurrence," BMC Cancer, vol. 13, article 182, 2013.

[30] O. M. Omran and H. S. Ata, "CD44s and CD44v6 in diagnosis and prognosis of human bladder cancer," Ultrastructural Pathol$o g y$, vol. 36, no. 3, pp. 145-152, 2012.

[31] A. M. Affy, S. Tate, B. Durbin-Johnson, D. M. Rocke, and T. Konia, "Expression of CD44s and CD44v6 in lung cancer and their correlation with prognostic factors," International Journal of Biological Markers, vol. 26, no. 1, pp. 50-57, 2011.

[32] M. Jijiwa, H. Demir, S. Gupta et al., "CD44V6 regulates growth of brain tumor stem cells partially through the AKT-mediated pathway," PLoS One, vol. 6, no. 9, Article ID e24217, 2011.

[33] C. W. Fan, L. Wen, Z. D. Qiang et al., "Prognostic significance of relevant markers of cancer stem cells in colorectal cancer-a meta analysis," Hepatogastroenterology, vol. 59, no. 117, pp. 14211427, 2012.

[34] R. Marhaba, M. Bourouba, and M. Zöller, "CD44v6 promotes proliferation by persisting activation of MAP kinases," Cellular Signalling, vol. 17, no. 8, pp. 961-973, 2005.

[35] M. Jijiwa, H. Demir, S. Gupta et al., "CD44V6 regulates growth of brain tumor stem cells partially through the AKT-mediated pathway," PLoS One, vol. 6, no. 9, Article ID e24217, 2011.

[36] K. Nakajima, K. Taniguchi, and K.-I. Mutoh, "Expression of CD44v6 as matrix-associated ectodomain in the bone development," Journal of Veterinary Medical Science, vol. 72, no. 8, pp. 1017-1022, 2010.

[37] V. Orian-Rousseau, "CD44, a therapeutic target for metastasising tumours," European Journal of Cancer, vol. 46, no. 7, pp. 1271-1277, 2010.

[38] S. Yang, H.-P. Wang, X.-Y. Wang et al., "Expression of CD44V6 in parotid pleomorphic adenoma and carcinoma ex pleomorphic adenoma," Expert Opinion on Investigational Drugs, vol. 19, supplement 1, pp. S101-S108, 2010.

[39] M. F. Spafford, J. Koeppe, Z. Pan, P. G. Archer, A. D. Meyers, and W. A. Franklin, "Correlation of tumor markers p53, bcl2, CD34, CD44H, CD44v6, and Ki-67 with survival and metastasis in laryngeal squamous cell carcinoma," Archives of Otolaryngology-Head and Neck Surgery, vol. 122, no. 6, pp. 627-632, 1996.

[40] B. Mack and O. Gires, "CD44s and CD44v6 expression in head and neck epithelia," PLoS One, vol. 3, no. 10, Article ID e3360, 2008.

[41] T. J. Heyse, D. Malcherczyk, R. Moll et al., "CD44: survival and metastasis in chondrosarcoma," Osteoarthritis and Cartilage, vol. 18, no. 6, pp. 849-856, 2010.

[42] H.-S. Kim, Y.-B. Park, J. H. Oh, J. Jeong, C. J. Kim, and S. H. Lee, "Expression of CD44 isoforms correlates with the metastatic potential of osteosarcoma," Clinical Orthopaedics and Related Research, no. 396, pp. 184-190, 2002.

[43] B. D. Gun, B. Bahadir, S. Bektas et al., "Clinicopathological significance of fascin and CD44v6 expression in endometrioid carcinoma," Diagnostic Pathology, vol. 7, article 80, 2012.

[44] F. J. Carmona, A. Villanueva, A. Vidal et al., "Epigenetic disruption of cadherin-11 in human cancer metastasis," The Journal of Pathology, vol. 228, no. 2, pp. 230-240, 2012.

[45] L. Li, J. Ying, H. Li et al., "The human cadherin 11 is a proapoptotic tumor suppressor modulating cell stemness through Wnt $/ \beta$-catenin signaling and silenced in common carcinomas," Oncogene, vol. 31, no. 34, pp. 3901-3912, 2012. 
[46] M. N. Marchong, C. Yurkowski, C. Ma, C. Spencer, S. Pajovic, and B. L. Gallie, "Cdh11 acts as a tumor suppressor in a murine retinoblastoma model by facilitating tumor cell death," PLoS Genetics, vol. 6, no. 4, Article ID e1000923, 2010.

[47] T. Kashima, J. Kawaguchi, S. Takeshita et al., "Anomalous cadherin expression in osteosarcoma: possible relationships to metastasis and morphogenesis," American Journal of Pathology, vol. 155, no. 5, pp. 1549-1555, 1999.

[48] T. Kashima, K. Nakamura, J. Kawaguchi et al., "Overexpression of cadherins suppresses pulmonary metastasis of osteosarcoma in vivo," International Journal of Cancer, vol. 104, no. 2, pp. 147154, 2003.

[49] S. I. Grivennikov, F. R. Greten, and M. Karin, "Immunity, Inflammation, and Cancer," Cell, vol. 140, no. 6, pp. 883-899, 2010.

[50] N. Warde, "Inflammation: cadherin 11: a key mediator of fibroblast inflammation," Nature Reviews Rheumatology, vol. 7, no. 7, p. 374, 2011.

[51] T. P. Hill, D. Später, M. M. Taketo, W. Birchmeier, and C. Hartmann, "Canonical Wnt $/ \beta$-catenin signaling prevents osteoblasts from differentiating into chondrocytes," Developmental Cell, vol. 8, no. 5, pp. 727-738, 2005.

[52] M. Kansara, M. Tsang, L. Kodjabachian et al., "Wnt inhibitory factor 1 is epigenetically silenced in human osteosarcoma, and targeted disruption accelerates osteosarcomagenesis in mice," Journal of Clinical Investigation, vol. 119, no. 4, pp. 837-851, 2009.

[53] L. Bongiovanni, F. Mazzocchetti, D. Malatesta et al., "Immunohistochemical investigation of cell cycle and apoptosis regulators (survivin, $\beta$-catenin, p53, caspase 3 ) in canine appendicular osteosarcoma," BMC Veterinary Research, vol. 8, article 78, 2012.

[54] F. Zhang, A. Chen, J. Chen, T. Yu, and F. Guo, "Influence of $\beta$ catenin small interfering RNA on human osteosarcoma cells," Journal of Huazhong University of Science and TechnologyMedical Science, vol. 31, no. 3, pp. 353-358, 2011.

[55] A. P. G. Guimarães, R. M. Rocha, I. W. da Cunha et al., "Prognostic impact of adenomatous polyposis coli gene expression in osteosarcoma of the extremities," European Journal of Cancer, vol. 46, no. 18, pp. 3307-3315, 2010.

[56] C. M. Piskun, A. Muthuswamy, M. K. Huelsmeyer, V. Thompson, and T. J. Stein, "Wnt/ $\beta$-catenin expression does not correlate with serum alkaline phosphatase concentration in canine osteosarcoma patients," PLoS One, vol. 6, no. 10, Article ID e26106, 2011.

[57] J. Deng, S. A. Miller, H.-Y. Wang et al., “ $\beta$-catenin interacts with and inhibits NF- $\kappa \mathrm{B}$ in human colon and breast cancer," Cancer Cell, vol. 2, no. 4, pp. 323-334, 2002.

[58] T. Masaki, A. Goto, M. Sugiyama et al., "Possible contribution of CD44 variant 6 and nuclear beta-catenin expression to the formation of budding tumor cells in patients with T1 colorectal carcinoma," Cancer, vol. 92, no. 10, pp. 2539-2546, 2001. 


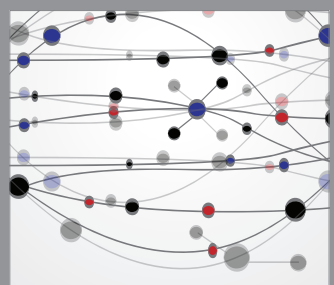

The Scientific World Journal
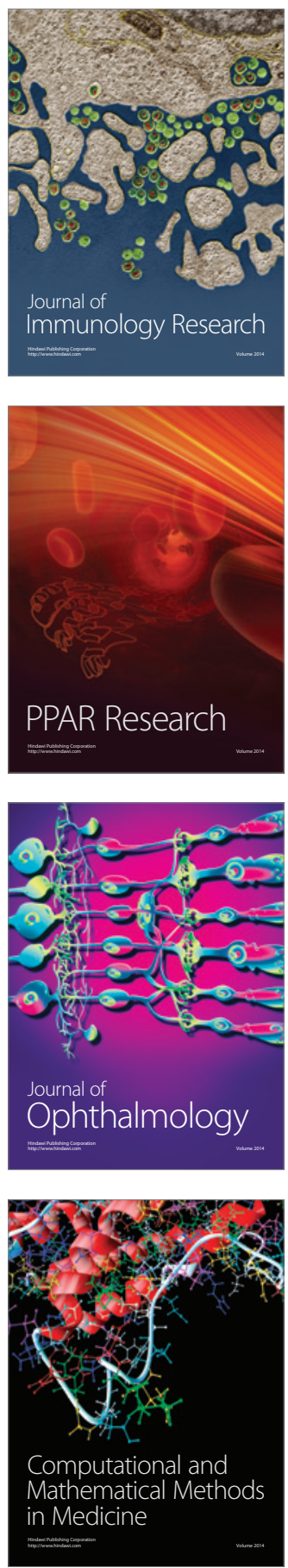

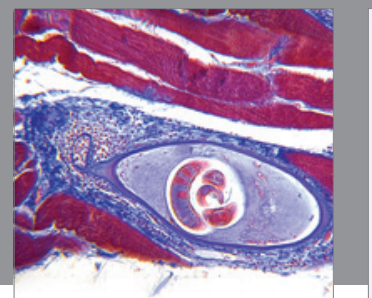

Gastroenterology

Research and Practice
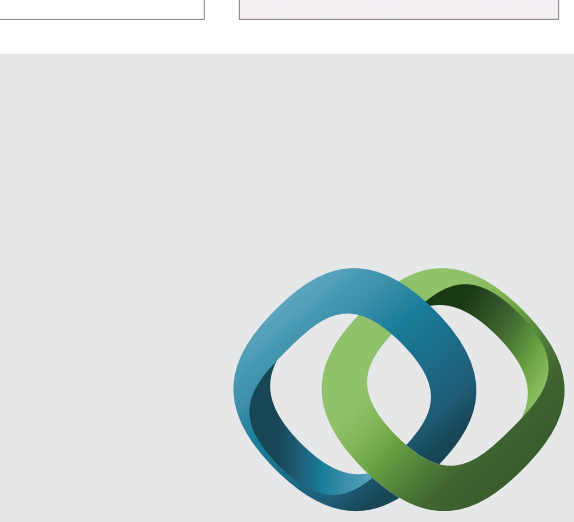

\section{Hindawi}

Submit your manuscripts at

http://www.hindawi.com
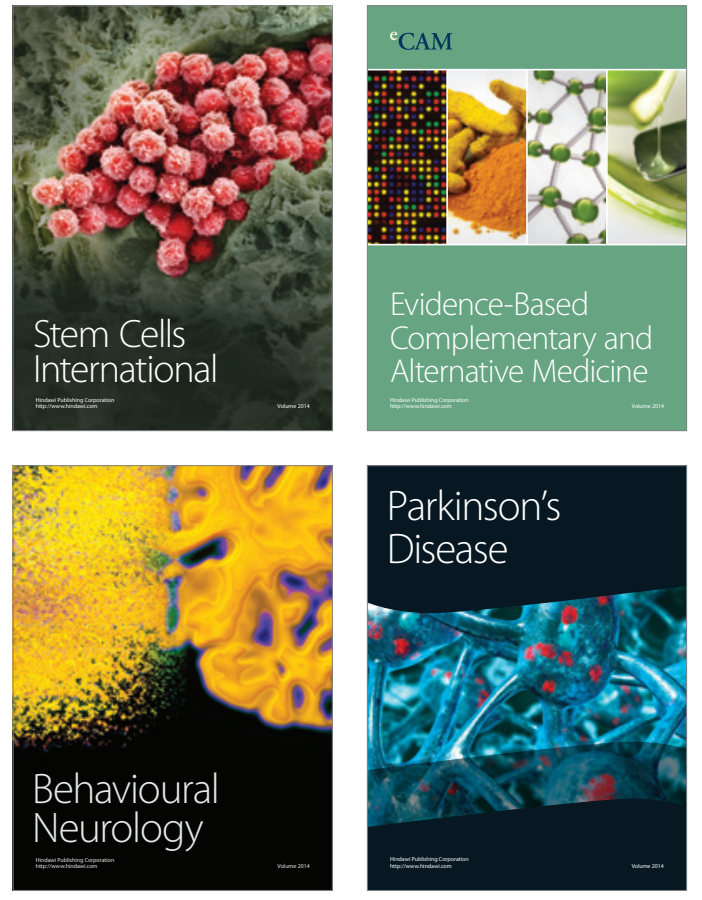
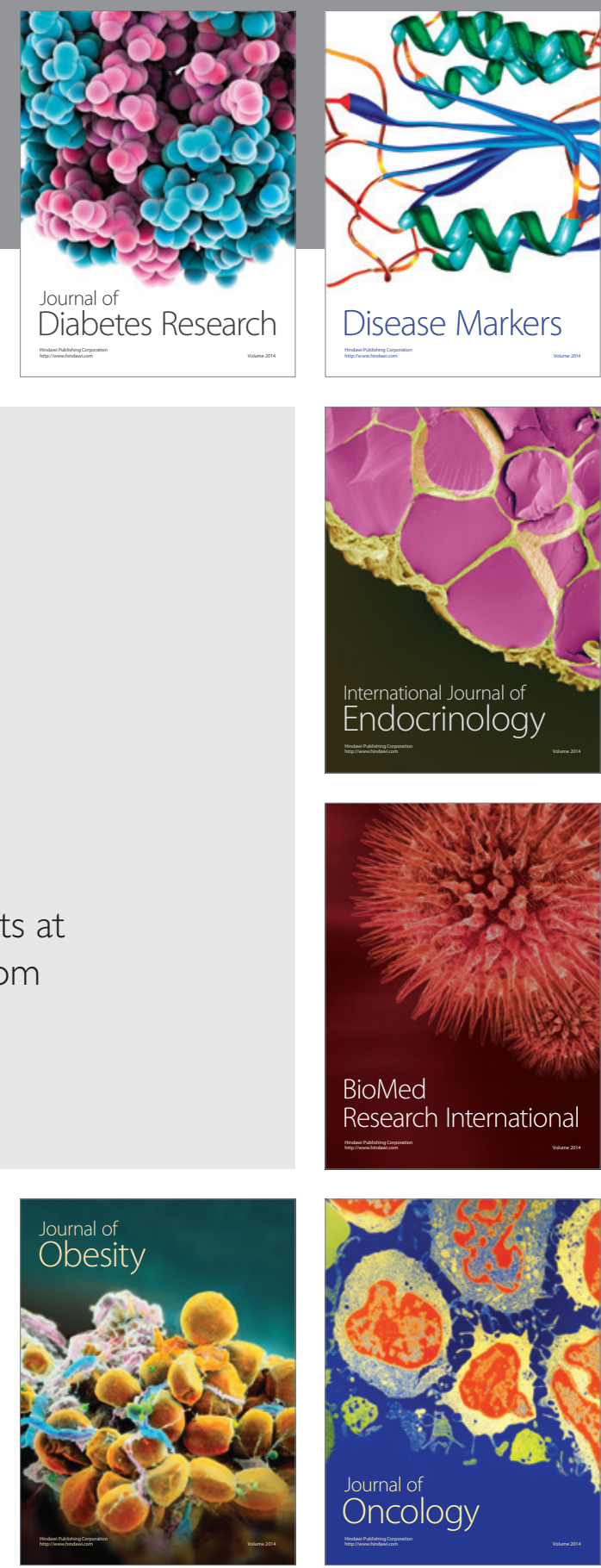

Disease Markers
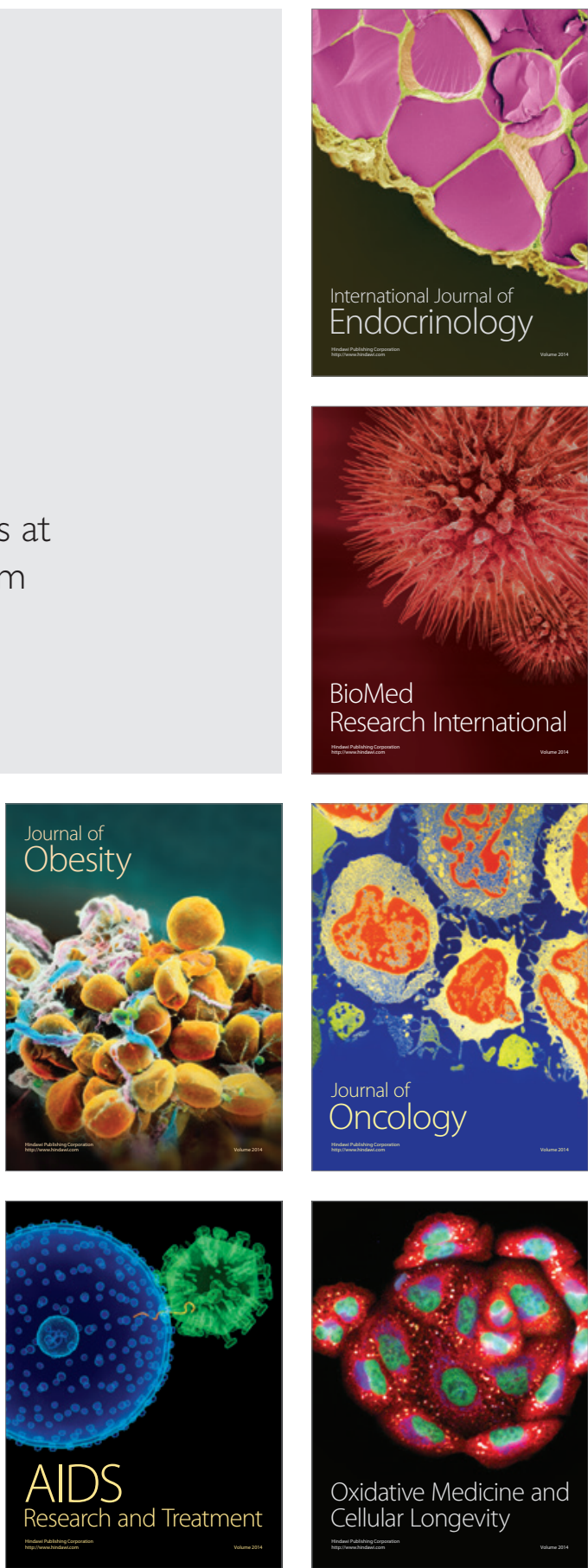